Leisure time physical activity and its relation to psychiatric comorbidities in depression. Findings from Finnish Depression and Metabolic Syndrome in Adults (FDMSA) study

Raatikainen, Ilkka

2019-12-01

Raatikainen , I, Mäntyselkä , P , Vanhala , M , Heinonen , A, Koponen , H , Kautiainen , H \& Korniloff , K 2019 , ' Leisure time physical activity and its relation to psychiatric comorbidities in depression. Findings from Finnish Depression and Metabolic Syndrome in Adults (FDMSA) study ' , Journal of Affective Disorders , vol. 259 , pp. 150-153 . https://doi.org/10.1016/j.jad.2019.08.039

http://hdl.handle.net/10138/320965

https://doi.org/10.1016/j.jad.2019.08.039

publishedVersion

Downloaded from Helda, University of Helsinki institutional repository.

This is an electronic reprint of the original article.

This reprint may differ from the original in pagination and typographic detail.

Please cite the original version. 
Short communication

\title{
Leisure time physical activity and its relation to psychiatric comorbidities in depression. Findings from Finnish Depression and Metabolic Syndrome in Adults (FDMSA) study
}

\author{
Ilkka Raatikainen $^{\mathrm{a}, \mathrm{b}, *}$, Pekka Mäntyselkä ${ }^{\mathrm{c}, \mathrm{d}}$, Mauno Vanhala ${ }^{\mathrm{c}}$, Ari Heinonen ${ }^{\mathrm{a}}$, Hannu Koponen ${ }^{\mathrm{e}}$ \\ Hannu Kautiainen ${ }^{\text {d,f }}$, Katariina Korniloff ${ }^{g}$ \\ ${ }^{\text {a } U n i v e r s i t y ~ o f ~ J y v a ̈ s k y l a ̈, ~ F a c u l t y ~ o f ~ S p o r t ~ a n d ~ H e a l t h ~ S c i e n c e s, ~ F i n l a n d ~}$ \\ ${ }^{\mathrm{b}}$ Assistive Technology Centre, Central Finland Health Care District, Jyväskylä, Finland \\ ${ }^{\mathrm{c}}$ Institute of Public Health and Clinical Nutrition, University of Eastern Finland, Kuopio, Finland \\ ${ }^{\mathrm{d}}$ Primary Health Care Unit, Kuopio University Hospital, Kuopio, Finland \\ ${ }^{e}$ Old Age Psychiatry, Department of Psychiatry, Helsinki University and Helsinki University Hospital, Psychiatry, Helsinki, Finland \\ ${ }_{\mathrm{f}}^{\mathrm{F}}$ Folkhälsan Research Center, Helsinki, Finland \\ ${ }^{\mathrm{g}}$ School of Health and Social Studies, JAMK University of Applied Sciences, Jyväskylä, Finland
}

A R T I C L E IN F O

\section{Keywords:}

Depression

Leisure time physical activity

Psychiatric comorbidities

\begin{abstract}
A B S R A C T
Purpose: The study aim was to examine association between leisure time physical activity (LTPA) and psychiatric comorbidities among people with depression.

Methods: Total 447 patients aged 35 years and older suffering from depressive symptoms (DS) and who were confirmed depression positive participated this study. The study was conducted between 2008 and 2011 in municipalities within Central Finland Hospital District. DS were determined with Beck Depression Inventory (BDI-21) with cutoff score $\geq 10$ and psychiatric diagnoses were confirmed by Mini-International Neuropsychiatric Interview (M.I.N.I.). LTPA, other diseases as well as use of antidepressant were captured by self-reported questionnaire. Participants also took part in physical examination. The associations between LTPA and psychiatric comorbidities were analyzed using generalized linear models.

Results: LTPA level was not related to number of psychiatric comorbidities (after adjustment for age, gender, BMI, BDI and use of antidepressant $p=0.24$ ) among depressed patients. The higher levels of LTPA were linearly associated with lower cardiovascular diseases $(p=0.036)$ and obesity $(p=0.006)$ as well as fewer DS $(p<0.001)$ among depressed patients.

Limitations: Possibility of LTPA level overestimation and study results generalizability to younger persons.

Conclusions: According to this study, LTPA level is not associated with psychiatric comorbidities among depressed patients in Finnish adult population. However, our results showed that the higher the LTPA level was, the less the participants suffered from depressive symptoms. In addition, higher levels of physical activity were associated with fewer heart diseases and obesity outlining the importance of overall health-care and health promotion although other forms of treatment are also needed.
\end{abstract}

\section{Introduction}

Depression is one of the most disability causing illnesses in the world (Whiteford et al., 2013) and its economical and individual burden are huge. Depression is often associated with one or more other psychiatric disorder and together they can impact one's life dramatically. Over $70 \%$ of depression diagnosed patients have at least one other psychiatric comorbidity (Kessler et al., 2003). Patient suffering from mental illnesses has over two-fold higher risk of early death compare to people in general and their life expectancy is ten years

\footnotetext{
Abbreviations: BDI (-21), Beck Depression Inventory; BMI, Body Mass Index; CVD, cardiovascular disease; DM, diabetes mellitus; DS, depressive symptoms; FDMSA-

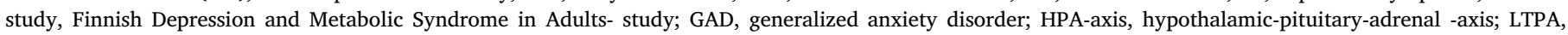

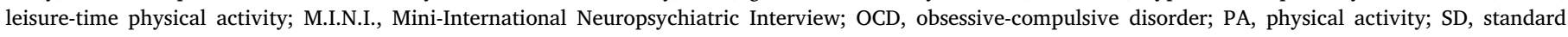
deviation; US, United States; vs, versus

* Corresponding author at: University of Jyväskylä, Faculty of Sport and Health Sciences, P.O.Box 35, FI-40014 Finland.

E-mail address: ilkka.t.raatikainen@student.jyu.fi (I. Raatikainen).
} 
shorter (Walker et al., 2015). Depression together with psychiatric comorbidities are found to increase the sensation of pain and decrease one's performance and quality of life (Bair et al., 2008). Depression is also associated with increased risk of many somatic diseases such as obesity, diabetes and cardiovascular diseases (Penninx et al., 2013).

According to recent knowledge leisure time physical activity (LTPA) is useful for treating and preventing depression. Physical activity reduces depressive symptoms (DS) in people with mental illness (Rosenbaum et al., 2014) and physically active individuals have lower risk for depression in the future compared to in-active ones (Mammen and Faulkner, 2013; Schuch et al., 2018). According to review of Mammen and Faulkner (2013) any physical activity (PA) level, including low levels (e.g., walking $<150 \mathrm{~min} /$ weeks), may prevent for future depression. Recent large United States (US) nationwide study (Chekroud et al., 2018) has revealed that physical exercised and physically active individuals had nearly $43 \%$ fewer poor mental health days than those who not in the past month. The relationship between PA and depression is also bidirectional, increased PA can reduce depressive symptoms as well as depression itself can reduce PA (Sieverdes et al., 2012).

As depression is often associated with multiple psychiatric comorbidities (Kessler et al., 2003), it is still largely unknown whether or how amount of LTPA is related to psychiatric comorbidities among depressive patients. Depression by itself and particularly with additional psychiatric comorbidities is not only individual misery but huge societal burden (Mrazek et al., 2014;Sobocki et al., 2006) and therefore it is sensible to figure out means and factors to resolve or at least reduce these negative effects of illness. Most of the previous studies investigating the relationship between depression and LTPA have not took psychiatric comorbidity into account or psychiatric comorbidities are excluded. Thus, the main aim of this study as a part of Finnish Depression and Metabolic Syndrome Study (FDMSA) is to explore the relationship between LTPA and psychiatric comorbidities among depressive diagnosed patients.

\section{Materials and methods}

\subsection{Participants}

The study data were drawn from the Finnish Depression and Metabolic Syndrome in Adults (FDMSA) study which was conducted between 2008 and 2011 in municipalities within Central Finland Hospital District with catchment area of 274000 inhabitants. The population of this study were enrolled from 35 years of age or older patients $(n=730)$ with depressive symptoms determined with Beck Depression Inventory (BDI-21) with cutoff score $\geq 10$. Participants were self-referred or referred by general practitioners to depression nurse case managers and who's psychiatric diagnoses were confirmed by diagnosis structured interview (M.I.N.I.) conducted by depression nurse case managers. Total 447 patients received a diagnosis of depression. The Ethics Committee of the Central Finland Hospital District approved the study protocol prior to the commencement of the study. All participants signed an informed written consent.

\subsection{Data sources}

All participants filled out a standard self-administered questionnaire containing questions about previously diagnosed somatic disorders, use of antidepressant, smoking habits, number of alcohol doses per week and leisure time physical activity (LTPA). Data on participants' socioeconomical background such as years of education, marital and employment status were also collected via questionnaire. Participants also took part on physical examination during the study visit. The physical examination included measurements of participants' weight and height during the study visit. Weight and height were measured in light clothing and was accurate to the nearest $0.5 \mathrm{~cm}$ and $0.1 \mathrm{~kg}$, respectively. The body mass index (BMI) was defined as person's weigth (body mass) divided by the square of the heigth. Obesity were defined when BMI $\geq 30$ (World Health Organization, 2018).

LTPA was assessed by asking:" How often do you do physical activity at least for half an hour so that you are out of breath and sweating?" Answers were classified into three levels: low (twice per month or less), moderate (once or twice per week), or high (three times per week or more). In previous studies self-assessed LTPA has shown a high correlation with physical fitness as measured by maximal oxygen uptake (Aires et al., 2003).

The severity of depressive symptoms (DS) was captured using the 21-item Beck Depression Inventory (BDI-21) (Beck et al., 1961), which was completed by the participants. The cut-off point was set to $\geq 10$ (Koponen et al., 2015). The psychiatric diagnosis and number of other psychiatric comorbidities were confirmed and obtained with a diagnostic Mini-International Neuropsychiatric Interview (M.I.N.I.) (Sheehan et al., 1998) delivered by trained study nurse.

\subsection{Statistical methods}

The results were presented as means with standard deviations (SD) or as counts with percentages. Statistical significance for the unadjusted analyses of linearity across the LTPA categories were evaluated using the Cochran-Armitage test, Cuzick test and analysis of variance with an appropriate contrast. Adjusted analyses of linearity (orthogonal polynomial) were evaluated using generalized linear models (e.g. analysis of co-variance, regression models and logistic models) with appropriate distribution and link function. Models included age, gender, years of education, marital status, comorbid diseases and household income as covariates. In the case of violation of the assumptions (e.g. non-normality), a bootstrap-type method was used (10 000 replications) to estimate standard errors. The normality of variables was evaluated by the Shapiro-Wilk W test. All analyses were performed using STATA 14.1.

\section{Results}

Table 1 shows the socio-demographic and clinical characteristics of the participants according to LTPA levels. BMI $(p=0.005)$ and depressive symptoms $(p<0.001)$, and further proportion of subjects with cardiovascular diseases $(p=0.036)$ and obesity $(p=0.006)$ decreased linearly across increasing LTPA categories. Although antidepressant was used by $68 \%(n=305)$ of participants, there were no linear association between the LTPA level and use of antidepressant $(p=0.53)$, nor with other clinical or socio-demographic characteristics either (Table 1).

The prevalence of psychiatric comorbidities among all depressed participants $(n=447)$ were as follow: generalized anxiety disorder (GAD) $56 \%$, suicidal $49 \%$, panic disorder $25 \%$, social phobia $16 \%$, alcohol abuse or dependence $15 \%$, hypomania $12 \%$, agoraphobia $6 \%$, posttraumatic stress reaction $6 \%$, obsessive-compulsive disorder (OCD) $6 \%$, bulimia or anorexia $2 \%$ and psychosis $2 \%$ (Table 2 ). Only one-fifth (23\%) of the participants had no psychiatric comorbidity. More than one third (38\%) of the participants had one comorbidity, $22 \%$ had two and $17 \%$ had at least three psychiatric comorbidities. Participants with one or more psychiatric comorbidities were younger (50 years vs. 53 years) and smoked more often ( $34 \%$ vs. $23 \%, p=0.023)$ than those without psychiatric comorbidities. Instead there were no difference in gender, BMI or educational years among participants with or without psychiatric comorbidities.

The statistical analysis revealed that LTPA level was not related to number of psychiatric comorbidities $(p=0.24)$. In addition, there was no significant linear relationship between the prevalence of psychiatric comorbidities and physical active level after adjusting the results for age, gender, BMI, BDI score and use of antidepressant (Table 2). 
Table 1

Depressed patients' socio-demographic and clinical characteristics at baseline.

\begin{tabular}{|c|c|c|c|c|c|}
\hline \multirow[t]{2}{*}{ Variables } & \multirow{2}{*}{$\begin{array}{l}\text { Total } \\
N=447\end{array}$} & \multicolumn{3}{|c|}{ Leisure time physical activity } & \multirow[t]{2}{*}{$P$-value* } \\
\hline & & $\begin{array}{l}\text { Low } \\
N=111\end{array}$ & $\begin{array}{l}\text { Moderate } \\
N=185\end{array}$ & $\begin{array}{l}\text { High } \\
N=151\end{array}$ & \\
\hline Female, n (\%) & $312(70)$ & $82(74)$ & $129(70)$ & $101(67)$ & 0.23 \\
\hline Age, years (SD) & $51(10)$ & $49(9)$ & $52(10)$ & $51(10)$ & 0.055 \\
\hline BMI, mean (SD) & $28.0(5.9)$ & $\begin{array}{l}28.9 \\
(6.3)\end{array}$ & $28.4(6.0)$ & $\begin{array}{l}26.9 \\
(5.2)\end{array}$ & 0.005 \\
\hline $\begin{array}{l}\text { Obesity, (BMI } \\
\quad \geq 30), \mathrm{n}(\%)\end{array}$ & $143(32)$ & 43 (39) & $65(35)$ & $35(23)$ & 0.006 \\
\hline $\begin{array}{l}\text { Education years } \\
\text { (SD) }\end{array}$ & $11.0(3.0)$ & $\begin{array}{l}11.0 \\
(2.9)\end{array}$ & $11.2(3.1)$ & $\begin{array}{l}10.8 \\
(3.1)\end{array}$ & 0.50 \\
\hline $\begin{array}{l}\text { Married or } \\
\text { cohabited, n } \\
(\%)\end{array}$ & $266(60)$ & $68(61)$ & $103(56)$ & $95(66)$ & 0.23 \\
\hline $\begin{array}{l}\text { Employment status, } \\
\text { n (\%) }\end{array}$ & & & & & 0.28 \\
\hline Employed & $202(45)$ & $55(50)$ & $84(45)$ & $63(42)$ & \\
\hline Unemployed & $113(25)$ & $32(29)$ & $41(22)$ & $40(26)$ & \\
\hline Student & $11(2)$ & $4(4)$ & $4(2)$ & $3(2)$ & \\
\hline Retired & $121(27)$ & $20(18)$ & $56(30)$ & $45(30)$ & \\
\hline Smoking, n (\%) & $142(32)$ & $47(42)$ & $49(26)$ & $46(30)$ & 0.067 \\
\hline $\begin{array}{l}\text { Alcohol, doses per } \\
\text { week, n (\%) }\end{array}$ & & & & & 0.99 \\
\hline None & $95(21)$ & $25(23)$ & 35 (19) & $35(23)$ & \\
\hline $1-5$ & $251(56)$ & $62(56)$ & $109(59)$ & $80(53)$ & \\
\hline $6-9$ & $41(9)$ & $6(5)$ & $19(10)$ & $16(11)$ & \\
\hline$\geq 10$ & $60(13)$ & $18(16)$ & $22(12)$ & 20 (13) & \\
\hline $\begin{array}{l}\text { BDI score, mean } \\
\text { (SD) }\end{array}$ & $23.4(8.2)$ & $\begin{array}{l}25.4 \\
(8.6)\end{array}$ & $23.4(8.2)$ & $\begin{array}{l}22.0 \\
(7.7)\end{array}$ & $<0.001$ \\
\hline $\begin{array}{l}\text { Antidepressant, } \mathrm{n} \\
\text { (\%) }\end{array}$ & $305(68)$ & $75(68)$ & $123(66)$ & $107(71)$ & 0.53 \\
\hline \multicolumn{6}{|l|}{ Disease, n (\%) } \\
\hline Neurological & $11(2)$ & $4(4)$ & $2(1)$ & $5(3)$ & 0.99 \\
\hline Cancer & $3(1)$ & $0(0)$ & $2(1)$ & $1(1)$ & 0.58 \\
\hline CVD & $73(16)$ & $10(9)$ & 34 (18) & 29 (19) & 0.036 \\
\hline Rheumatic & $15(3)$ & $4(4)$ & $9(5)$ & $2(1)$ & 0.25 \\
\hline Lung & $42(9)$ & $10(9)$ & $12(6)$ & $20(13)$ & 0.18 \\
\hline DM & 64 (14) & $18(16)$ & $23(12)$ & 23 (15) & 0.90 \\
\hline
\end{tabular}

BDI beck depression inventory, CVD cardiovascular disease, DM diabetes mellitus.

${ }^{*} \mathrm{p}$ for linearity across the LTPA categories.

\section{Discussion}

\subsection{Main findings and study implications}

Our findings suggested that LTPA is not associated with psychiatric comorbidities in patients with depressive symptoms. However, as in line with some earlier findings (Barengo et al., 2017; Korniloff et al., 2010) our study showed that LTPA had positive association with such health-related factors as DS obesity and CVD in depressive patients. This is important, since it has been shown in recent review that people with severe mental illness with low LTPA has increased risk of cardiovascular disease and premature mortality (Vancampfort et al., 2017).

Because PA, in general, has positive effect on mental health, it would be expected that these positive effects of PA are reflected on psychiatric comorbidities as well. Although, for example Bartley et al. (2013) have concluded in their meta-analysis that current evidence does not support the use of aerobic exercise as an effective treatment for anxiety disorders as compared to other treatment. In our study, the majority of study participants suffered from GAD (56\%) and as PA seems to be useful in depression it may have lesser impact on depression with anxiety disorder (Bartley et al., 2013). This could be one reason why LTPA does not seem to have positive association with psychiatric comorbidities in this study.

There are also neurobiological changes associated with depression e.g. in hypothalamic-pituitary-adrenal (HPA)- axis homeostasis, neurotransmitters, neurogenesis and inflammatory markers where PA could work as a "scaffolding" factor that support amelioration processes (Archer et al., 2014). Again, neurotic disorders might "purely" associate more with "mind" than "body". This could also explain, at least partly, why positive health effects of PA associated more clearly with pure depression but not depression with additional psychiatric disorders and that PA can reduce depressive symptoms but not necessarily psychiatric comorbidities.

Recent study of Chekroud et al. (2018) has also revealed that while physical exercise is in general useful for mental health, more exercise is not always better and that the optimal amount lies between 30 and $60 \mathrm{~min}$ per day three to five times a week. As depression itself can reduce PA (Sieverdes et al., 2012), neurotic disorders might force one to move more and therefore neutralize depressions effects on PA. This phenomenon might be reflected in our study also because we were not able to catch those who exercise "more". Nor we did not use objective PA measurement instead of subjective assessment nor we didn't know

Table 2

Psychiatric comorbidities among depressed patients according to leisure-time physical activity level at baseline.

\begin{tabular}{|c|c|c|c|c|c|}
\hline Variables & $\begin{array}{l}\text { Total } \\
N=447\end{array}$ & \multicolumn{3}{|c|}{ Leisure time physical activity } & $P$ for linearity" \\
\hline GAD, n (\%) & $250(56)$ & $68(61)$ & $101(54)$ & $81(54)$ & 0.62 \\
\hline Panic disorder, n (\%) & $111(25)$ & $26(23)$ & $46(25)$ & $39(26)$ & 0.24 \\
\hline Alcohol abuse or dependence, $\mathrm{n}(\%)$ & $65(15)$ & $17(15)$ & $25(14)$ & $23(15)$ & 0.93 \\
\hline Social phobia, n (\%) & $71(16)$ & $20(18)$ & $30(16)$ & $21(14)$ & 0.83 \\
\hline Hypomania, n (\%) & $55(12)$ & $16(14)$ & $17(9)$ & $22(15)$ & 0.57 \\
\hline OCD, n (\%) & $26(6)$ & $8(7)$ & $7(4)$ & $11(7)$ & 0.45 \\
\hline Bulimia or anorexia, $n(\%)$ & $11(2)$ & $7(6)$ & $1(1)$ & $3(2)$ & 0.68 \\
\hline Psychosis, n (\%) & $7(2)$ & $3(3)$ & $2(1)$ & $2(1)$ & 0.69 \\
\hline $\begin{array}{l}\text { Number of psychiatric comorbidities, mean (SD) } \\
\text { Number } n(\%)\end{array}$ & $1.5(1.3)$ & $1.6(1.4)$ & $1.4(1.2)$ & $1.4(1.2)$ & 0.24 \\
\hline 0 & $102(23)$ & $23(21)$ & $44(24)$ & $35(23)$ & \\
\hline 1 & $169(38)$ & $37(33)$ & $77(42)$ & $55(36)$ & \\
\hline
\end{tabular}

GAD generalized anxiety disorder, OCD obsessive-compulsive disorder.

${ }^{*} \mathrm{p}$ for linearity across the LTPA categories, adjusted for gender, age, BMI, BDI and use of antidepressant. 
what kind of PA lifestyle each participant had before the study due to cross sectional study design. This may have affected to these results also.

With these study findings without more extensive investigation we cannot say what is the relevance of LTPA in depression in terms of psychiatric comorbidities. While these findings can increase and widen our knowledge about factors related to depression, it also raises questions about methods and tools which are suitable in prevention and treatment of illness. As PA has many health benefits in depression it's suitability as treatment method to reduce psychiatric comorbidities associated with depression are arguable. Therefore, further research and more knowledge from the underlying factors behind these study findings are needed.

\subsection{Strengths and limitations}

The main strength of this study is the use of diagnostic interview (M.I.N.I.) to confirm depression and psychiatric comorbidities. Another strength of this study is national representative study population with catchment area of 274000 inhabitants. Main limitation lies on the selfreported LTPA which may prone to overestimation by participants. Although, a self-reported questionnaire has proven to be a valid and reliable method to measure PA in cross-sectional studies (Aires et al., 2003) and in this study design we were not trying to estimate exact amount and intensity of PA instead of crude LTPA level categorization. Moreover, generalization of study results to younger persons cannot be done due to study population of 35 and older in this study. Also, generalizability to other countries might be questionable as in Finnish society social equity is in very high level and for example physical education and health promotion as well as health services are well organized compared many other countries.

\section{Conclusion(s)}

LTPA level was not associated with psychiatric comorbidities among depressive diagnosed patients. This may suggest a different pathophysiology in depression and anxiety disorders as the level of LTPA was inversely associated with depressive symptoms. However, higher levels of physical activity associated with fewer heart diseases and obesity highlights the importance of LTPA promotion in the treatment of comorbid depression although other forms of treatment for comorbid anxiety disorders are also needed.

\section{Funding}

This research did not receive any specific grant from funding agencies in the public, commercial, or not-for-profit sectors.

\section{CRediT authorship contribution statement}

Ilkka Raatikainen: Writing - original draft. Pekka Mäntyselkä: Conceptualization, Methodology, Project administration, Writing - review \& editing. Mauno Vanhala: Conceptualization, Methodology, Writing - review \& editing. Ari Heinonen: Supervision, Writing - review \& editing. Hannu Koponen: Conceptualization, Methodology, Writing - review \& editing. Hannu Kautiainen: Data curation, Formal analysis, Software, Writing - review \& editing. Katariina Korniloff: Conceptualization, Methodology, Writing - review \& editing.

\section{Declaration of Competing Interest}

The authors declare that they have no conflict of interest.

\section{Acknowledgments}

The authors would like to thank the depression nurse case managers who took part in the practical implementation of the FDMSA: Mari Alanko, Harri Back, Timo Hannula, Anu Holopainen, Ritva Häkkinen, Katja Johansson, Eija Kinnunen, Kaija Luoma, Hannele Niemi, Hillevi Peura, Inga Pöntiö, Kirsi Rouvinen, Tiina Silvennoinen and Marianne Vihtamäki; the FDMSA study nurses Anne Kirmanen, Seija Torkkeli, Reetta Oksanen and Olli Niemi, and Pia Jauhiainen, scientific secretary of the study.

\section{Supplementary materials}

Supplementary material associated with this article can be found, in the online version, at doi:10.1016/j.jad.2019.08.039.

\section{References}

Aires, N., Selmer, R., Thelle, D., 2003. The validity of self-reported leisure time physical activity, and its relationship to serum cholesterol, blood pressure and body mass index. A population based study of 332,182 men and women aged 40-42 years. Eur. J. Epidemiol. 18 (6), 479-485.

Archer, T., Josefsson, T., Lindwall, M., 2014. Effects of physical exercise on depressive symptoms and biomarkers in depression. CNS. Neurol. Disord.Drug. Targets 13 (10), $1640-1653$.

Bair, M.J., Wu, J., Damush, T.M., Sutherland, J.M., Kroenke, K., 2008. Association of depression and anxiety alone and in combination with chronic musculoskeletal pain in primary care patients. Psychosom. Med. 70 (8), 890-897. https://doi.org/10.1097/PSY. 0 b013e318185c510.

Barengo, N.C., Antikainen, R., Borodulin, K., Harald, K., Jousilahti, P., 2017. Leisure-time physical activity reduces total and cardiovascular mortality and cardiovascular disease incidence in older adults. J. Am. Geriatr. Soc. 65 (3), 504-510.

Bartley, C.A., Hay, M., Bloch, M.H., 2013. Meta-analysis: aerobic exercise for the treatment of anxiety disorders. Prog. Neuro-Psychopharmacol. Biol. Psychiatry 45, 34-39.

Beck, A.T., Ward, C.H., Mendelson, M., Mock, J., Erbaugh, J., 1961. An inventory for measuring depression. Arch. Gen. Psychiatry 4, 561-571.

Chekroud, S.R., Gueorguieva, R., Zheutlin, A.B., Paulus, M., Krumholz, H.M., Krystal, J.H., Chekroud, A.M., 2018. Association between physical exercise and mental health in $1 \cdot 2$ million individuals in the USA between 2011 and 2015: a cross-sectional study. Lancet Psychiatry 5 (9), 739-746.

Kessler, R.C., Berglund, P., Demler, O., Jin, R., Koretz, D., Merikangas, K.R., ..., Wang, P.S., 2003. The epidemiology of major depressive disorder: results from the national comorbidity survey replication (NCS-R). JAMA 289 (23), 3095-3105.

Koponen, H., Kautiainen, H., Leppänen, E., Mäntyselkä, P., Vanhala, M., 2015. Association between suicidal behaviour and impaired glucose metabolism in depressive disorders. BMC Psychiatry 15 (1), 163.

Korniloff, K., Hakkinen, A., Kautiainen, H., Koponen, H., Peltonen, M., Mantyselka, P., ..., Vanhala, M., 2010. Leisure-time physical activity and metabolic syndrome plus depressive symptoms in the FIN-D2D survey. Prev. Med. 51 (6), 466-470. https://doi.org/10.1016/j ypmed.2010.09.007.

Penninx, B.W., Milaneschi, Y., Lamers, F., Vogelzangs, N., 2013. Understanding the somatic consequences of depression: Biological mechanisms and the role of depression symptom profile. BMC Medicine 11, 129 .

Mammen, G., Faulkner, G, 2013. Physical activity and the prevention of depression: a systematic review of prospective studies. Am. J. Prev. Med. 45 (5), 649-657.

Mrazek, D.A., Hornberger, J.C., Altar, C.A., Degtiar, I., 2014. A review of the clinical, economic, and societal burden of treatment-resistant depression: 1996-2013. Psychiatric Serv. 65 (8), 977-987. https://doi.org/10.1176/appi.ps.201300059.

Rosenbaum, S., Tiedemann, A., Sherrington, C., Curtis, J., Ward, P.B., 2014. Physical activity interventions for people with mental illness. J. Clin. Psychiatry 75 (09), 964-974. https:// doi.org/10.4088/JCP.13r08765.

Schuch, F.B., Vancampfort, D., Firth, J., Rosenbaum, S., Ward, P.B., Silva, E.S., ..., Stubbs, B., 2018. Physical activity and incident depression: a meta-analysis of prospective cohort studies. Am. J. Psychiatry 175 (7), 631-648. https://doi.org/10.1176/appi.ajp.2018. 17111194.

Sheehan, D.V., Lecrubier, Y., Sheehan, K.H., Amorim, P., Janavs, J., Weiller, E., ..., Dunbar, G.C., 1998. The mini-international neuropsychiatric interview (MINI): the development and validation of a structured diagnostic psychiatric interview for DSM-IV and ICD-10. J Clin. Psychiatry 59, 22-23.

Sieverdes, J.C., Ray, B.M., Sui, X., Lee, D., Hand, G.A., Baruth, M., Blair, S.N., 2012. Association between leisure time physical activity and depressive symptoms in men. Med. Sci. Sports Exerc. 44 (2), 260-265. https://doi.org/10.1249/MSS.0b013e31822e00a6.

Sobocki, P., Jonsson, B., Angst, J., Rehnberg, C., 2006. Cost of depression in Europe. J. Mental Health Policy Econ. 9 (2), 87-98. Retrieved from. http://ovidsp.ovid.com/ovidweb.cgi? $\mathrm{T}=\mathrm{JS} \& \mathrm{PAGE}=$ reference $\& \mathrm{D}=$ emed7\&NEWS $=\mathrm{N} \& \mathrm{AN}=2006325880$.

Vancampfort, D., Firth, J., Schuch, F.B., Rosenbaum, S., Mugisha, J., Hallgren, M., ..., De Hert, M., 2017. Sedentary behavior and physical activity levels in people with schizophrenia, bipolar disorder and major depressive disorder: a global systematic review and meta-analysis. World Psychiatry 16 (3), 308-315.

Walker, E.R., McGee, R.E., Druss, B.G., 2015. Mortality in mental disorders and global disease burden implications: a systematic review and meta-analysis. JAMA Psychiatry 72 (4), 334-341.

Whiteford, H.A., Degenhardt, L., Rehm, J., Baxter, A.J., Ferrari, A.J., Erskine, H.E., .... Johns, N., 2013. Global burden of disease attributable to mental and substance use disorders: findings from the global burden of disease study 2010. The Lancet 382 (9904), 1575-1586. World Health Organization. (2018). Obesity and overweight. (2018). Retrieved fromhttp:// www.who.int/news-room/fact-sheets/detail/obesity-and-overweight. 